\title{
Surveying the TeV Sky with Milagro
}

\author{
C.P. Lansdell for the Milagro Collaboration \\ University of Maryland, Dept. of Physics, College Park, MD 20742 \\ Los Alamos National Laboratory, MS H803, Los Alamos, NM 87545
}

\begin{abstract}
A wide field of view, high duty factor, $\mathrm{TeV}$ gamma-ray observatory is essential for studying $\mathrm{TeV}$ astrophysical sources, because most of these sources are either highly variable or are extended. Milagro is such a $\mathrm{TeV}$ detector and has performed the deepest survey of the Northern Hemisphere sky. In addition to detecting the known TeV sources of the Crab Nebula and Markarian 421, Milagro has made the first detection of diffuse $\mathrm{TeV}$ emission from the Galactic plane. The Milagro data has been searched for unknown point sources and extended sources. Evidence for a new extended $\mathrm{TeV}$ source is seen and is coincident with an EGRET unidentified source. The Milagro data has also been searched for the predicted $\mathrm{TeV}$ emitters of gamma-ray bursts, galaxy clusters, and EGRET unidentified sources. Based on the success of Milagro, a second generation water Cherenkov gamma-ray observatory is planned which will give an increase in sensitivity of more than an order of magnitude.
\end{abstract}

Keywords: gamma-ray astronomy; extensive air-shower detectors; cosmic-ray detectors PACS: 95.55.Ka; 95.85.Pw; 29.40.Ka

\section{MILAGRO}

Milagro [1] is a water-Cherenkov detector at an altitude of $2650 \mathrm{~m}$ capable of continuously monitoring the overhead sky and is composed of a central $60 \mathrm{~m} \times 80 \mathrm{~m}$ pond with a sparse $200 \mathrm{~m} \times 200 \mathrm{~m}$ array of 175 "outrigger" tanks surrounding it. The pond is instrumented with two layers of photomultiplier tubes. The top "air-shower" layer consists of 450 PMTs under $1.4 \mathrm{~m}$ of purified water while the bottom "muon" layer has 273 PMTs located $5 \mathrm{~m}$ below the surface. The air-shower layer allows the accurate measurement of shower particle arrival times used for direction reconstruction and triggering. The greater depth of the muon layer is used to detect penetrating muons and hadrons. Simple cuts have been developed to distinguish between gamma-ray- and hadron/muon-induced showers [2]. The outrigger array improves the core location and angular resolution of the detector by providing a longer lever arm with which to reconstruct events. The angular resolution improves from $\approx 0.75^{\circ}$ to $\approx 0.45^{\circ}$ when outriggers are used in the reconstruction.

Milagro's large field of view ( $\sim 2 \mathrm{sr})$ and high duty cycle $(>90 \%)$ allow it to monitor the entire overhead sky continuously, making it well-suited to searching for new $\mathrm{TeV}$ sources and scanning known sources at higher energies.

\section{OBSERVATIONS}

The Crab Nebula acts as Milagro's standard candle due to its long-term, unchanging energy emission through many wavelengths. With 1.5 years of data where outriggers 
are part of the reconstruction, the Crab is seen at 9.7 $\sigma$. This is compared to $10 \sigma$ in 4.5 years of pre-outrigger data. Other $\mathrm{TeV}$ point sources seen in the same 4.5 years of data include Markarian 421 at 5.4 $\sigma$ and a hot spot in the Cygnus region of the Galaxy at $5.9 \sigma$.

\section{Extended Source Survey}

Milagro has the opportunity to search for extended or diffuse sources of $\mathrm{TeV}$ emission by increasing the bin size when surveying the sky. The data are scanned from $2.1^{\circ}$ to $5.9^{\circ}$ in $0.2^{\circ}$ steps. At $5.9^{\circ}$, the Cygnus region of the galaxy becomes the most luminous source of $\mathrm{TeV}$ gamma rays in the Northern sky with a $9.1 \sigma$ excess. An ensemble of simulated sky maps is used to compute the probability of observing an excess as improbable as the observed $9.1 \sigma$ from any position in the Northern sky with any of the bin sizes searched. The probability of such an excess occurring as a result of statistical fluctuations is $2 \times 10^{-14}$.

Convolving the Cygnus region data with Milagro's point spread function $\left(0.75^{\circ}\right)$ reveals a resolvable structure which does not seem to agree in the finer details with the known matter distribution of that area as seen by EGRET [3]. This suggests the existence of extended sources or multiple point sources. Two of the seven EGRET unidentified sources in this area (3EG J2021+3716 and 3EG J2016+3657) coincide spatially with the position of the maximum $\mathrm{TeV}$ gamma-ray excess seen by Milagro. The flux at the maximum point is estimated to be $500 \mathrm{mCrab}$.

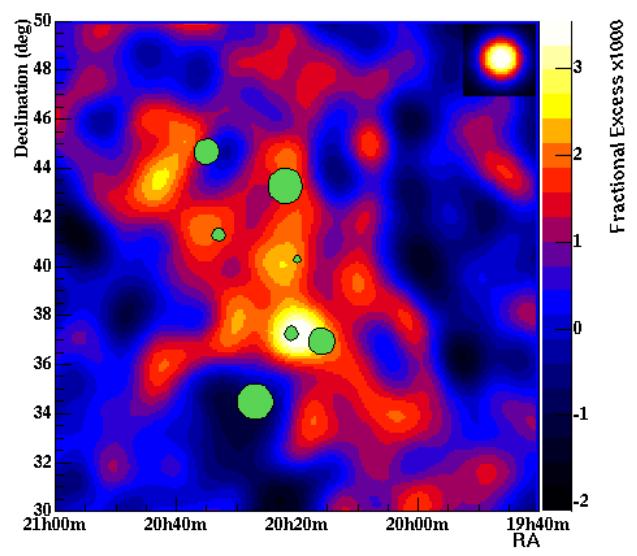

FIGURE 1. Fractional event excess distribution in the Cygnus region. The circles are the positions of seven EGRET unidentified sources detected in the region. The size of the circles indicates the 95\% EGRET position errors.

\section{Galactic Plane Survey}

EGRET data shows an excess in emission from the Galactic plane above $1 \mathrm{GeV}$ that is not clearly explained by known gamma-ray production mechanisms [3]. An examination at $\mathrm{TeV}$ energies by Milagro shows emission from the Galactic plane at $7.5 \sigma$ in 4.5 years of data. Towards the Galactic center, a $-2.61 \pm 0.03$ (stat.) \pm 0.05 (sys.) spectrum is found while towards the Galactic anti-center, an upper limit of $-2.66(99 \%$ $\mathrm{CL}$ ) is observed [4]. The Milagro $\mathrm{TeV}$ spectral indices are consistent with an 
extrapolation of the highest energy EGRET points to within one standard deviation, though the outer galaxy measurement suggests a softer spectrum than seen in the inner galaxy.

\section{GRB Survey}

Milagro has not observed TeV emission from a GRB yet, though there is evidence for VHE emission from GRB 970417a, which was seen in the prototype detector Milagrito [5, 6]. Milagro can calculate upper limits on TeV emission from GRBs, though the process is highly model-dependent [7,8]. With the launch of Swift, the number of GRBs within Milagro's field of view is expected to increase to 20 GRBs per year. This is compared to $\sim 4$ per year within the field of view prior to Swift.

\section{FUTURE DETECTOR - HAWC}

Based on the success of Milagro and the lessons learned with the water-Cherenkov technique to observe air-showers, a proposal will be submitted to construct a new land-based detector called HAWC (High Altitude Water Cherenkov experiment). HAWC will be one large pond $(300 \mathrm{~m} \times 300 \mathrm{~m})$ at a higher elevation than Milagro $5200 \mathrm{~m}$ is considered here.

Monte Carlo simulations show that HAWC can obtain sensitivity improvements of up to $\sim 60$ times that of Milagro. The Crab Nebula can be detected at the $5 \sigma$ level within minutes, compared to more than 4.5 months to see the Crab at the same level in Milagro. In one year of running, HAWC will see the Galactic plane at $\sim 60 \sigma$, compared to $3.5 \sigma$ in one year of Milagro running. HAWC will have similar instantaneous sensitivity as air-Cherenkov telescopes like Whipple, but with the added benefit of being able to scan the entire overhead sky continuously.

\section{ACKNOWLEDGMENTS}

We acknowledge Scott Delay and Michael Schneider for their dedicated efforts in the construction and maintenance of the Milagro experiment. This work has been supported by the National Science Foundation, the US Department of Energy, Los Alamos National Laboratory, the University of California, and the Institute of Geophysics and Planetary Physics.

\section{REFERENCES}

1. Atkins, R,. et al., Nucl. Instr. Meth., A449, 478 (2000).

2. Atkins, R., et al., Astrophys. J., 595, 803 (2003).

3. Hunter, S.D., et al., Astrophys. J., 481, 205 (1997).

4. Atkins, R., et al., "Evidence for TeV Gamma-ray Emission from a Region of the Galactic Plane", Phys. Rev. Lett., to be published.

5. Atkins, R., et al., Astrophys. J. Lett., 533, L119 (2000).

6. Atkins, R., et al., Astrophys. J., 583, 824 (2003).

7. Atkins, R., et al., Astrophys. J. Lett., 604, L25 (2004).

8. Atkins, R., et al., "Constraints on Very High Energy Gamma-Ray Emission from Gamma-Ray Bursts", Astrophys. J., to be published. 